BNL-112503-2016-JA

\title{
Novel Trends in Pair Distribution Function Approaches on Bulk Systems with Nanoscale Heterogeneities
}

Emil S. Bozin and Simon J. L. Billinge

Submitted to Neutron News

July 2016

\author{
Condensed Matter Physics and Material Science Department \\ Brookhaven National Laboratory
}

\author{
U.S. Department of Energy \\ USDOE Office of Science (SC), \\ Basic Energy Sciences (BES) (SC-22)
}

\footnotetext{
Notice: This manuscript has been authored by employees of Brookhaven Science Associates, LLC under Contract No. DE-SC0012704 with the U.S. Department of Energy. The publisher by accepting the manuscript for publication acknowledges that the United States Government retains a non-exclusive, paid-up, irrevocable, world-wide license to publish or reproduce the published form of this manuscript, or allow others to do so, for United States Government purposes.
} 


\section{DISCLAIMER}

This report was prepared as an account of work sponsored by an agency of the United States Government. Neither the United States Government nor any agency thereof, nor any of their employees, nor any of their contractors, subcontractors, or their employees, makes any warranty, express or implied, or assumes any legal liability or responsibility for the accuracy, completeness, or any third party's use or the results of such use of any information, apparatus, product, or process disclosed, or represents that its use would not infringe privately owned rights. Reference herein to any specific commercial product, process, or service by trade name, trademark, manufacturer, or otherwise, does not necessarily constitute or imply its endorsement, recommendation, or favoring by the United States Government or any agency thereof or its contractors or subcontractors. The views and opinions of authors expressed herein do not necessarily state or reflect those of the United States Government or any agency thereof. 


\section{Scientific Report: Novel Trends in Pair Distribution Function Approaches on Bulk Systems with Nanoscale Heterogeneities}

Novel materials for high performance applications increasingly exhibit structural order on the nanometer length scale; a domain where crystallography, the basis of Rietveld refinement, fails [1]. In such instances the total scattering approach, which treats Bragg and diffuse scattering on an equal basis, is a powerful approach. In recent years, the analysis of the total scattering data became an invaluable tool and the gold standard for studying nanocrystalline, nanoporous, and disordered crystalline materials. The data may be analyzed in reciprocal space directly, or Fourier transformed to the real-space atomic pair distribution function (PDF) and this intuitive function examined for local structural information. Here we give a number of illustrative examples, for convenience picked from our own work, of recent developments and applications of total scattering and PDF analysis to novel complex materials. There are many other wonderful examples from the work of others.

The total scattering structure function, $F(Q)$ (where $Q$ is the momentum transfer) is obtained from a powder diffraction pattern by correcting for experimental aberrations, normalizing and dividing by the atomic form factor. The atomic pair distribution function (PDF), $G(r)$ (where $r$ is the interatomic distance) is simply a sine Fourier transform of $F(Q)$. Since $F(Q)$ contains both Bragg and diffuse scattering, G(r) yields the direct-space information of atomic arrangements on various length scales [2] from atomic near neighbors, through nanoscale to bulk length-scales. With the advent of amazing advances in modern experimental instrumentation [3, 4] as well as in data reduction [5] and modeling software $[6,7]$, the applicability and scope of the PDF method has become extensive.

With length-scale reduction to nanometer size, materials display novel or enhanced size-tunable properties compared to their bulk counterparts, with a broad range of promising applications such as nanosensors, biomedical imaging, optoelectronic devices, energy materials, and catalysts. In this domain, the presumption of a periodic solid, which is the basis of a crystallographic analysis, breaks down entirely. Since the PDF probes atomic correlations without regard of translational symmetry, it is well suited for nanoscale materials characterization and provides a unique perspective to the nanostructure problem.[1] It allows access to a wealth of information: accurate determination of atomic-scale structure, homogeneous and inhomogeneous strain, structural defects, geometrical particle parameters such as the particle diameter and shape, all important for understanding the fundamental mechanisms and processes in novel nanostructured materials.

The past decade has brought a particularly important breakthrough for application of the PDF approach, with utilization of novel experimental setups such as an image-plate detector coupled with high energy synchrotron x-ray radiation [3] and high flux - high data throughput neutron instruments. [4] This has resulted in a dramatic reduction of the data collection time by multiple orders of magnitude, with simultaneous access to a high momentum transfer range and maintaining excellent counting statistics. This not only enabled high data throughput experiments, but also opened up new avenues for systematic studies of atomic structure on a nanoscale not possible before, such as with challenging sample sizes and restricted experimental geometries including in situ [8] and under extreme conditions.[9] High throughput setups enable measurements with excellent counting statistics, hence allowing weak scattering or dilute features to be investigated. This is particularly advantageous for differential PDF methods, through which relatively weak contributions to the total PDF (a few wt\% or less), such as arising from adsorbed guest species, are studied. Important material classes to which PDF is applicable encompass diverse problems including energy conversion/storage/efficiency, ionic conduction, and so on. 
While, without a doubt, the advancements of the PDF methodology have revolutionized studies of nanoscale and nanoporous systems, their role in the better understanding of conventional bulk materials should by no means be neglected. With that in mind, this overview aims at highlighting some of the recent trends in so-called small-box PDF modeling approach to characterize short-range fluctuations in bulk materials. The overview brings three examples that illustrate the extents to which the nanoscale atomic structure studies can be pushed and how they impact our understanding of the material's properties. In bulk materials, the PDF allows assessment and comparison of structural information on different length scales simultaneously from the same measurement providing a self-consistent framework for doing so. This is of particular importance for understanding the properties of materials with a nanoscale structure that observably differs from the crystallographic average structure. This might seem to be a rather odd or unique occurrence, though these total scattering tools are showing us that the effect is actually quite widespread in materials.

A textbook example of such a case is presented by a neutron PDF study of nanometer length-scale fluctuations in $\mathrm{La}_{1-\mathrm{x}} \mathrm{Ca}_{\mathrm{x}} \mathrm{MnO}_{3}$ (Figure 1). This system exhibits an extremely rich phase diagram over a wide range of $\mathrm{Ca}$ concentration and temperature and is a host to the famous colossal magnetoresistance effect and metal-to-insulator phase transition (MIT) at which material transforms from the ferromagnetic metallic (FM) to the paramagnetic insulating (PI) state on heating. [10] Atomic PDF profiles, such as these shown in Figure 1a for $\mathrm{LaMnO}_{3}$, reveal important local structural fluctuations. Notably in this case, important observations of the nanoscale structural fluctuations can already be made by a simple comparison of experimental PDF data below and above long-range orbital ordering temperature and associated Jahn-Teller distortions of $\mathrm{MnO}_{6}$ octahedra. Although the average structure appears "regular" for the range of concentrations displaying the MIT (Figure 1b), local fluctuations are present in the material that mark themselves in the Rietveld refinement through an unphysical increase of the atomic displacement parameters upon crossing into the insulating regime. The PDF small-box modeling approach applied over a variable $r$-range to over 100 neutron PDF datasets and some 13 compositions provides invaluable insight into the nature of these fluctuations (Figure 1c), characterized by local distortions of the $\mathrm{MnO}_{6}$ octahedra that decrease from about $0.23 \AA$ in the undoped endmember to smaller values with increased $\mathrm{Ca}$ content in the insulating phase, rectifying an earlier understanding of the material and setting the limits for theoretical considerations. Once onset in the insulating regime, these fluctuations are recently found to persist up to the highest temperatures, deep into the rhombohedral phase where they are crystallographically strictly prohibited (Figure 1d and 1e).[11] Their spatial correlations are estimated from small-box modeling to be of the order of a nanometer only. Physical properties, in this case MIT, appear to closely resemble the local, rather than the average structural behavior, emphasizing the importance of the PDF approach in this case.

In another material system in the strongly-correlated electron physics arena, hole-doped high temperature superconductor $\mathrm{La}_{2-x} \mathrm{Ba}_{\mathrm{x}} \mathrm{CuO}_{4}$, the complexity in properties exposes itself through interplay of competing orders - including charge ordering (in form of stripes) and superconductivity. When there is long range stripe order in the copper-oxygen planes, bulk superconductivity is suppressed. These layered perovskite-like materials display a sequence of structural phases (in the order of appearance on cooling: high temperature tetragonal - HTT, low temperature orthorhombic LTO, and low temperature tetragonal - LTT). It was previously found that for $x=0.125$ the stripe phase forms and is stabilized by the LTT structural regime at base temperature or at elevated pressure, where the in-plane $\mathrm{Cu}-\mathrm{O}$ bonds are orthogonally inequivalent (OI) with bond difference within a single $\mathrm{CuO}_{4}$ plaquette being subtle $0.005 \AA$. Upon heating the structure transforms first to LTO, and then further to HTT average structure. In both the in-plane $\mathrm{Cu}-\mathrm{O}$ bonds become orthogonally equivalent (OE) and the average $\mathrm{Cu}-\mathrm{O}$ bond disparity disappears. Interestingly however, a recent neutron PDF study based on over 150 datasets and 4 compositions [12] that explored the evolution of the local structure for several 
Ba compositions, revealed that there is fluctuating local OI state persisting to temperatures way above the LTT-LTO phase boundary (Figure 2), allowing for a possibility of there being charge-order fluctuations at temperatures above the ordering temperature $\mathrm{T}_{\mathrm{CO}}$ that is coincidental with the LTT/LTO transformation. As one moves away from $\mathrm{x}=0.125$ composition, the size of the distortions compatible with long range stripe order decreases, and bulk superconductivity recovers. It is important to note here that the standard PDF measurements do not discriminate between the elastic and inelastic scattering channels, and therefore do not provide information on the dynamics of underlying local structural distortions. Complementary inelastic neutron scattering (INS) measurements were done that not only confirm the existence of the local LTT distortions, but also reveal their dynamic character. Both neutron PDF and INS consistently suggest that the correlation length of these distortions is very short, of the order of just 5-7 Å.[12]

As a final example, we look at the thiospinel $\mathrm{CuIr}_{2} \mathrm{~S}_{4}$ system which exhibits a first-order metal-toinsulator transition on cooling, coincidental with the average symmetry lowering from cubic to triclinic, simultaneous charge and orbital ordering, and $5 \mathrm{~d}^{5}$ Ir spin dimerization. A dramatic $0.5 \AA$ shortening of Ir-Ir dimer distances accompanies the low- $\mathrm{T}$ ordered insulating state providing a uniquely strong structural fingerprint of the dimers. Upon doping a small amount of $\mathrm{Cr}$ on the Ir site the MIT is quickly destabilized and, albeit with the doped system retaining cubic average symmetry, it exhibits semi-metallic behavior. Due to the appreciable neutron absorption of Ir, in this case an x-ray based PDF approach was more appropriate. An early PDF study of $\mathrm{CuIr}_{2} \mathrm{~S}_{4}$, based solely on a data comparison and no modeling involved, established unquestionably that no local dimers survive into the high temperature metallic regime. However, a more recent PDF study of the $(x, T)$ phase diagram, based on some 900 x-ray datasets and 16 Cr-compositions (Figure 3),[13] revealed that although the long range dimer order is quickly suppressed by doping, the dimers do survive locally at low temperature, only to disappear on heating in very much the same fashion as the long-range ordered ones do in $\mathrm{CuIr}_{2} \mathrm{~S}_{4}$ endmember. Due to the large structural distortion size associated with the dimers, their presence and spatial extent can be readily observed from the discrepancies of the cubic model fits to the data seen in the difference curve (Figure 3b), and nicely contrasted to the case when intentionally wrong cubic model is attempted on a dataset displaying long range dimer order. Surprisingly, the electric transport properties displayed in ( $x, T)$ diagrammatic way (Figure 3e) resemble the nanoscale structure phase diagram (Figure 3d), rather than the average structure one (Figure 3c), again pointing to the impact of the nanoscale structure on the electronic properties in this bulk system. The PDF study further reveals that the origin behind the persistence of the local Ir-Ir dimers at low temperature (or rather their re-entrant behavior as it turns out) can be tracked down to Cr-doping induced strain.[13]

High throughput neutron and x-ray total scattering measurements, coupled with new PDF modeling software capable of handling a large volume of data, provide an opportunity to probe the structure of materials on different length scales with an unprecedented level of detail. These studies include the evolution of local fluctuations across phase diagrams, allowing for important observations to be made systematically, consistently, and in "one go", giving completely new insights into why materials exhibit the properties that they do. The synergy of high data throughput instruments and high- performance computing capabilities brings studies of local fluctuations in complex bulk materials and exploration of their relevance for the displayed properties to a completely new level, allowing comprehensive nanoscale structure surveys and enabling novel insights that were unavailable before.

\section{By Emil S. Bozin and Simon J. L. Billinge}

E.S.B. (Brookhaven National Laboratory) bozin@bnl.gov 
S.J.L.B. (Columbia University, Brookhaven National Laboratory)

sb2896@columbia.edu

\section{References:}

[1] S.J.L. Billinge, I. Levin, Science 316, 561 (2007).

[2] T. Egami, S.J.L. Billinge, Underneath the Bragg Peaks: Structural Analysis of Complex Materials (2nd Ed., Elsevier, Amsterdam, 2012).

[3] P.J. Chupas, X. Qiu, J.C. Hanson, P.L. Lee, C.P. Grey and S.J.L. Billinge, J. Appl. Crystallogr. 36, 1342-1347 (2003).

[4] J. Neuefeind, M. Feygenson, J. Carruth, R. Hoffmann, K. Chipley, Nuclear Instruments and Methods in Physics Research B 287, 68-75 (2012).

[5] X. Yang, P. Juhás, C. Farrow and S.J.L. Billinge, arXiv 1402.3163 (2015).

[6] C.L. Farrow, P. Juhás, J. Liu, D. Bryndin, E.S. Bozin, J. Bloch, Th. Proffen and S.J.L. Billinge, J. Phys: Condens. Mat. 19, 335219 (2007).

[7] P. Juhás, C.L. Farrow, X. Yang, K.R. Knox and S.J.L. Billinge, Acta Cryst. A 71, 562 (2015).

[8] P.J. Chupas, K.W. Chapman, P.L. Lee, J. Appl. Cryst. 40, 463 (2007).

[9]. L. Ehm, S.M. Antao, J. Chen, D.R. Locke, F.M. Michel, C.D. Martin, T. Yu, J.B. Parise, P.L. Lee, P.J. Chupas, S.D. Shastri, Q. Guo, Powder Diffr. 22, 108 (2007).

[10] E.S. Bozin, M. Schmidt, A.J. DeConinck, G. Paglia, J.F. Mitchell, T. Chatterji, P.G. Radaelli, Th. Proffen, S.J.L. Billinge, Phys. Rev. Lett. 98, 137203 (2007).

[11] M. Shatnawi, E.S. Bozin, J.F. Mitchel, S.J.L. Billinge, Phys. Rev. B 93, 165138 (2016).

[12] E.S. Bozin, R. Zhong, K.R. Knox, G. Gu, J.P. Hill, J.M. Tranquada and S.J.L. Billinge, Phys. Rev. B 91, 054521 (2015).

[13] E.S. Bozin, K.R. Knox, P. Juhás, Y.S. Hor, J.F. Mitchell and S.J.L. Billinge, Sci. Rep. 4, 4081 (2014).

\section{Figures:}

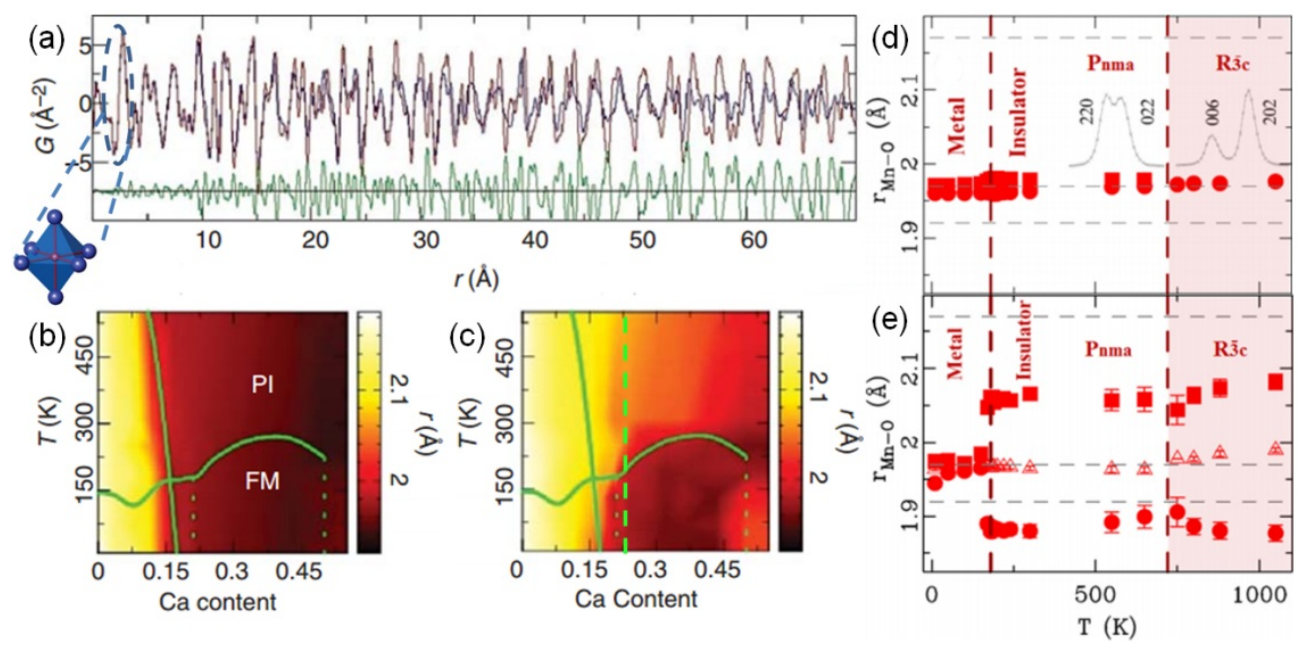

Figure 1: PDF reveals a different local structure in $\mathrm{Ca}$ doped $\mathrm{LaMnO}_{3}$ due to a local Jahn-Teller distortion of the $\mathrm{MnO}_{6}$ octahedral unit, comprising four shorter and two long $\mathrm{Mn}-\mathrm{O}$ bonds. (a) Experimental neutron PDFs for $700 \mathrm{~K}$ (blue) and $750 \mathrm{~K}$ (red) bracing the orbital order-to-disorder Jahn-Teller transition that occurs at $720 \mathrm{~K}$ in $\mathrm{LaMnO}_{3}$. The difference curve (green) reveals that the distortion persists locally in the crystallographically undistorted high-temperature phase, and that 
structure over a $\sim 1 \mathrm{~nm}$ length scale remains unchanged. Parametric evolution of (b) the length of the average long $\mathrm{Mn}-\mathrm{O}$ bond across the phase diagram of $\mathrm{La}_{1-\mathrm{x}} \mathrm{Ca}_{\mathrm{x}} \mathrm{MnO}_{3}$ from Rietveld refinements and (c) of the length of the local long $\mathrm{Mn}-\mathrm{O}$ bond from short-range PDF refinement. PI, paramagnetic insulating phase; FM, ferromagnetic metallic phase. [10] Vertical dashed line denotes $x=0.22$ composition, for which evolution of (d) average and (e) local Mn-O octahedral distances is shown up to very high temperature and display very different behaviors.[11]
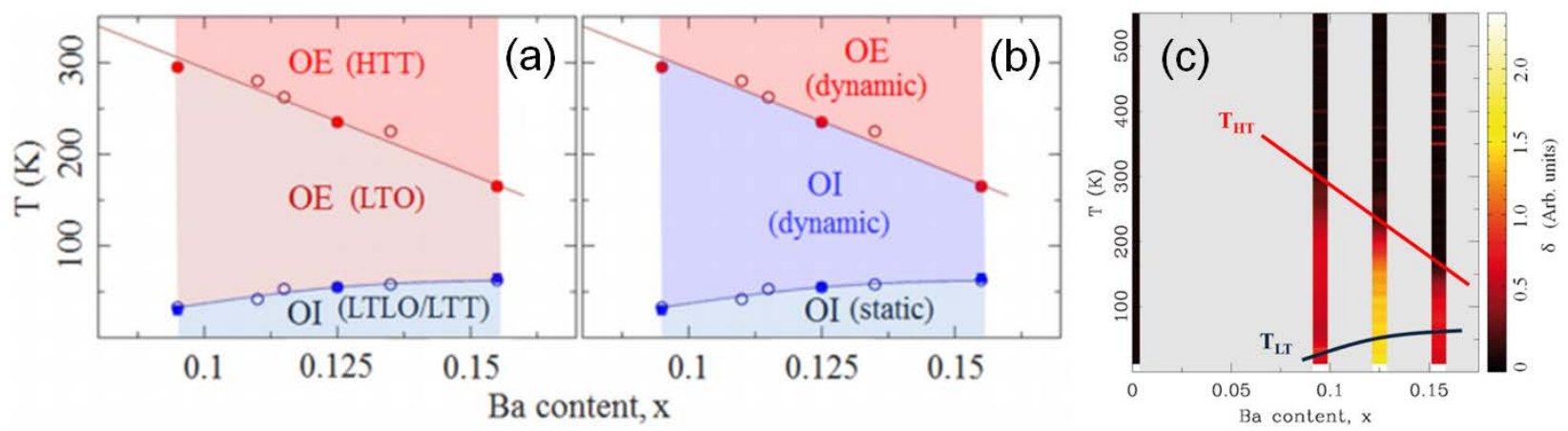

Figure 2: $\mathrm{PDF}$ reveals to existence of local distorted $\mathrm{CuO} 4$ placquets in the high temperature superconductor $\mathrm{La}_{2-\mathrm{x}} \mathrm{Ba}_{\mathrm{x}} \mathrm{CuO}_{4}$, (a) Average structure $(\mathrm{x}, \mathrm{T})$ phase diagram of $\mathrm{La}_{2-\mathrm{x}} \mathrm{Ba}_{\mathrm{x}} \mathrm{CuO}_{4}$ which has been replotted to highlight whether the structure is OE or OI. Shaded are regions explored in the study that have average OE (HTT and LTO) and OI (LTLO/LTT) symmetries, as indicated. (b) Local structure phase diagram with phase designations based on the results of combined PDF and INS study. (c) False-color diagram of $(\mathrm{x}, \mathrm{T})$ evolution of $\delta$, PDF-derived parameter assigned as a measure of the OI-ness of the local structure. Solid lines mark structural transition temperature: $\mathrm{T}_{\mathrm{HT}}$ (HTT/LTO) and $\mathrm{T}_{\mathrm{LT}}$ (LTO/LTT).[12]
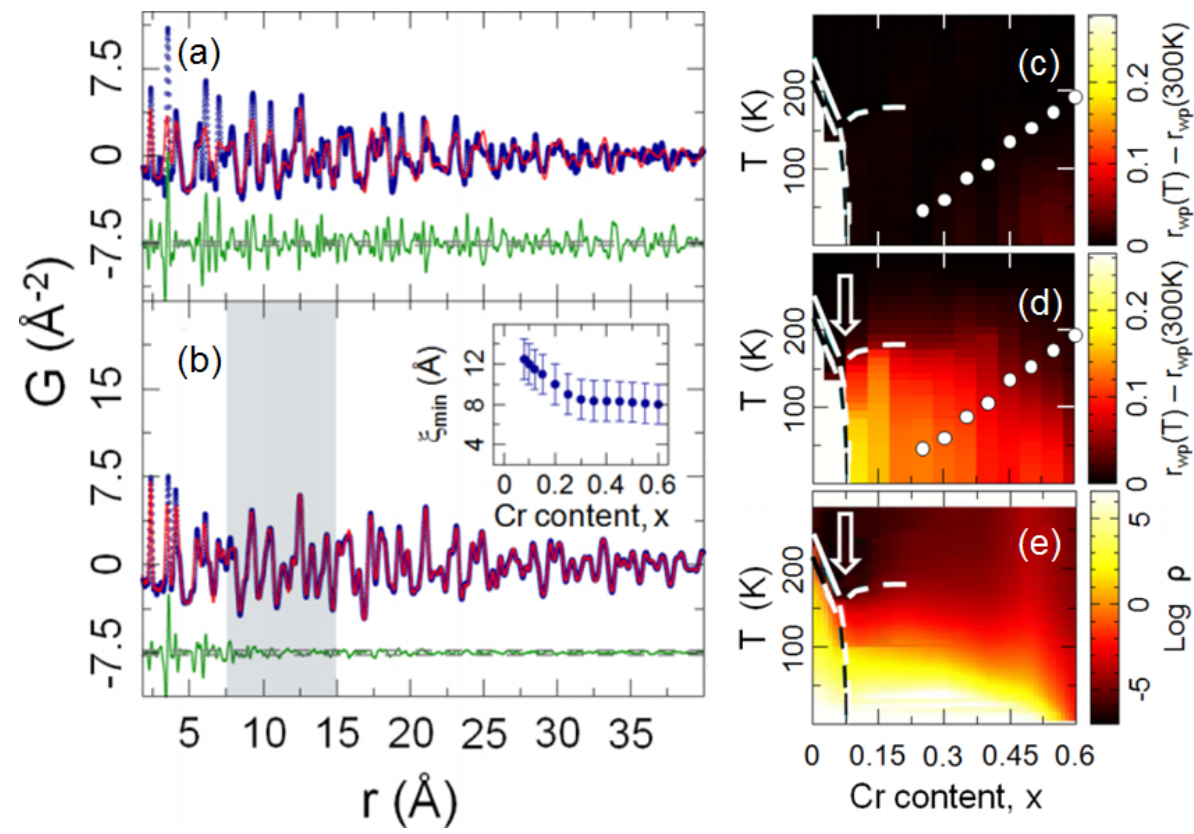

Figure 3: PDF can determine whether local Ir-Ir dimers exist at different places in the the phase diagram of $\mathrm{Cr}$ doped $\mathrm{CuIr}_{2} \mathrm{~S}_{4}$ giving new insights into the metal-insulator transition in this material. (a) $\mathrm{CuIr}_{2} \mathrm{~S}_{4}$ data at $10 \mathrm{~K}$ (open blue symbols), the best fit cubic model (solid red line), and the difference curve (green solid line) offset for clarity. Dashed lines are experimental uncertainties on the $2 \sigma$ level. (b) Same as (a) but for $40 \% \mathrm{Cr}$ sample. The grey area marks the r-region where the crossover from 
local to average behavior occurs. Inset shows estimated short-range dimer order correlation length for various $\mathrm{Cr}$ concentrations at base temperature. Structural phase diagrams as seen from the perspective of the cubic model differential fit residual for (c) average and (d) local structures respectively. (e) (x,T) phase diagram as seen from the perspective of the electric transport property.[13]

Address: $\quad$ Condensed Matter Physics and Materials Science Department

Brookhaven National Laboratory

734 Brookhaven Avenue

Upton, NY 11973

USA

Applied Physics and Applied Mathematics Department

Columbia University

500 W. 120th Street

New York, NY 10027

USA 